\title{
Polyacrylamide-Clay Nanocomposite Hydrogels: Rheological and Light Scattering Characterization
}

\author{
Oguz Okay*,† and Wilhelm Oppermann*,* \\ Department of Chemistry, Istanbul Technical University, 34469 Maslak, Istanbul, Turkey, and Institute \\ of Physical Chemistry, Clausthal University of Technology, Arnold-Sommerfeld-Str. 4, \\ 38678 Clausthal-Zellerfeld, Germany
}

Received December 21, 2006; Revised Manuscript Received February 19, 2007

\begin{abstract}
The free-radical polymerization of acrylamide in aqueous clay dispersions and the structure of the resulting polymer-clay nanocomposite hydrogels have been investigated by rheometry using oscillatory deformation tests. Laponite was used as clay particles in the hydrogel preparation. The reactions were carried out with and without the presence of the chemical cross-linker $N, N^{\prime}$-methylenebis(acrylamide) (BAAm). In the absence of BAAm, increasing clay concentration from 0.2 to $7 \%$ results in 3 orders of magnitude increase of the elastic modulus $G^{\prime}$ of the hydrogels. At a clay concentration of $5 \%$ or above, all the reaction systems, with or without BAAm, exhibit similar elastic moduli, indicating that clay mainly determines the rubber elasticity of the hydrogels. The loss factor $\tan \delta$ was found to be around 0.1 , indicating that the nanocomposite hydrogels are much more viscous than the conventional hydrogels. Increasing the amount of clay also increases the viscous, energy dissipating properties of the nanocomposite hydrogels, which are responsible for their improved mechanical properties. Dynamics of the nanocomposite hydrogels was also investigated by dynamic light scattering. The ensembleaveraged scattered intensity of the hydrogels varies nonmonotonically with the clay concentration due to the action of clay both as a cross-linker and as an ionic component during the formation of the nanocomposite hydrogels.
\end{abstract}

\section{Introduction}

Polymer hydrogels have an important role in many technological areas such as drug delivery, sensors, and superabsorbent materials. ${ }^{1}$ In these applications, precise information on the molecular constitution of the hydrogels as well as their elastic properties is required. Hydrogels are usually prepared by freeradical copolymerization of acrylamide (AAm)-based monomers with a chemical cross-linker such as $N, N^{\prime}$-methylenebis(acrylamide) (BAAm) in an aqueous solution. Because of the presence of a solvent (water) during the gel formation process, polymer hydrogels are usually soft and fragile when handled in the swollen state.

Recently, Haraguchi et al. prepared hydrogels with improved mechanical properties starting from AAm-based monomers together with Laponite as a physical cross-linker, replacing the traditional chemical cross-linkers. ${ }^{2-5}$ Laponite, a synthetic hectorite clay, when suspended in water forms disklike particles with a thickness of $1 \mathrm{~nm}$, a diameter of about $25 \mathrm{~nm}$, and a negative surface charge density of $0.014 e^{-1} / A^{2}$, which stabilizes dispersions in water. Formation of a cross-linked polymer network using a small amount of Laponite indicates that these nanoparticles act as a multifunctional cross-linker with a large effective functionality. The action of Laponite as a cross-linker seems to be due to strong interactions at the clay-polymer interface and the combination of polymer and inorganic components within a single material on a nanoscale level. Such nanocomposite hydrogels are attractive for a wide range of applications. ${ }^{6,7}$

With regard to the characterization of the microstructure of hydrogels, scattering methods such as light scattering, smallangle X-ray scattering (SAXS), and small-angle neutron scattering (SANS) were used in the past two decades. ${ }^{8-11}$ It was

\footnotetext{
$\dagger$ Technical University Istanbul.

$\doteqdot$ Clausthal University of Technology.
}

shown that the scattering intensity from polymer gels is always larger than that from the solution of the same polymer at the same concentration. The excess scattering over the scattering from polymer solution is related to the inhomogeneous distribution of the polymer material along the gel sample, which is called spatial gel inhomogeneity. ${ }^{12,13}$ An inhomogeneous gel consists of regions of high polymer concentration (less swollen) surrounded by regions of low polymer concentration (highly swollen). Inhomogeneity is a usual feature of gels formed in a fairly dilute state. It is particularly strong in gels formed by free-radical copolymerization. ${ }^{14-16}$ This is due to the fact that at the beginning of such polymerizations extensive cyclization and multiple cross-linking reactions take place, leading to rather compact clusters or microgel particles. As the reaction proceeds to higher conversion, these objects get interconnected to form a continuous network. Thus, the concentration fluctuations are "frozen" in the final network. Static light scattering measurements indicate a correlation length of $10^{1} \mathrm{~nm}$ for all the AAmbased hydrogels. ${ }^{15,17}$ In poly( $N$-isopropylacrylamide) (PNIPA)/ Laponite nanocomposite hydrogels, however, two levels of organization characterized by two correlation lengths were observed. ${ }^{18}$ The smaller correlation length is of the same order of magnitude $\left(10^{1} \mathrm{~nm}\right)$ as that observed in chemically crosslinked hydrogels. The longer correlation length around 200$250 \mathrm{~nm}$ seems to be peculiar to cross-linking via nanoparticles. ${ }^{18}$

It was also shown that, contrary to the conventional chemically cross-linked hydrogels, the nanocomposite hydrogels formed using Laponite as a cross-linker exhibit extraordinary mechanical toughness, tensile moduli, and tensile strengths. These quantities rise with increasing Laponite content. ${ }^{2-5}$ For example, PNIPA/Laponite hydrogels are much tougher and deformable by a factor of 20-50 in deformability and of 3001000 in toughness in comparison with conventional PNIPA hydrogels. While most studies were made with NIPA, we used AAm as the monomer. Our preliminary experiments also showed that, compared to the weak and fragile hydrogels based 
on AAm and BAAm, the hydrogels derived from AAm and Laponite are very tough and could withstand high levels of elongation without damage. Haraguchi explained this unusual feature of Laponite hydrogels with the uniform distribution of the clay particles within the hydrogel sample. ${ }^{2-4}$ Thus, the network chains in such hydrogels are uniform and long compared to conventional hydrogels, this fact being responsible for their improved mechanical properties. However, many model networks prepared from monodisperse polymer chains do not exhibit such distinct features. ${ }^{13}$ Shibayama et al. investigated the structure and molecular dynamics of the nanocomposite hydrogels by SAXS, X-ray scattering, and dynamic light scattering (DLS). ${ }^{19}$ Their results show that the chain dynamics of nanocomposite hydrogels is similar to the conventional chemically cross-linked gels. Nie et al. demonstrated by DLS measurements that $\sim 90 \%$ of the total scattering intensity from PNIPA/Laponite hydrogels corresponds to the frozen concentration fluctuations. ${ }^{20}$ These results also show that the nanocomposite hydrogels are inhomogeneous like the conventional hydrogels. Contrary to these findings, Shibayama et al. recently showed by contrast-matched SANS experiments that the inhomogeneity in these hydrogels is mainly due to the clay-clay and the clay-polymer scatterings. ${ }^{21,22}$ They also suggested that the action of Laponite as a multifunctional cross-linker is responsible for the good mechanical performance of such materials. Thus, the reason for the extraordinary mechanical properties of nanocomposite hydrogels is still a discussion point.

In the present work, we monitored the gelation reactions of AAm and Laponite in aqueous solutions by rheometry using oscillatory deformation tests. To our knowledge, no rheological investigation has yet been conducted for this kind of nanocomposite hydrogels. To understand the effect of Laponite as a crosslinking agent, the gelation reactions were carried out with and without the presence of the chemical cross-linker BAAm. The complex shear modulus $G^{*}$ measured can be resolved into its real and imaginary components, i.e.

$$
G^{*}=G^{\prime}+i G^{\prime \prime}
$$

where the elastic (or storage) modulus $G^{\prime}$ is a measure of the reversibly stored deformation energy and the viscous (or loss) modulus $G^{\prime \prime}$ represents a measure of the irreversibly dissipated energy during one cycle. As will be seen below, the loss factor $\tan \delta=G^{\prime \prime} / G^{\prime}$ is much larger for the nanocomposite hydrogels in comparison with conventional polyacrylamide (PAAm) hydrogels. This feature of the nanocomposite hydrogels seems to be responsible for their improved mechanical properties. It was also of inherent interest to characterize the microstructure of the hydrogels by the light scattering technique. DLS measurements were carried out at various Laponite and BAAm concentrations, and the degree of the spatial gel inhomogeneities was determined.

\section{Experimental Part}

Materials. Acrylamide (AAm, Merck), $N, N^{\prime}$-methylenebis(acrylamide) (BAAm, Merck), ammonium persulfate (APS, Merck), and $N, N, N^{\prime}, N^{\prime}$-tetramethylethylenediamine (TEMED, Merck) were used as received. The synthetic hectorite clay, Laponite RDS $\left(\mathrm{Na}^{+}{ }_{0.7}\left[\mathrm{Si}_{8} \mathrm{Mg}_{5.5} \mathrm{Li}_{0.3}\right) \mathrm{O}_{20}(\mathrm{OH})_{4}\right]^{0.7-}$, modified with pyrophosphate ions $\left(\mathrm{P}_{2} \mathrm{O}_{7}{ }^{4-}\right)$ ) was provided by Rockwood Ltd. Suspensions of Laponite RDS were prepared by dispersing the white powder at the preset concentrations in deionized water with vigorous stirring for 1 week. Stock solutions of APS and TEMED were prepared by dissolving $0.2 \mathrm{~g}$ of APS and $0.625 \mathrm{~mL}$ of TEMED each in $25 \mathrm{~mL}$ of distilled water. Stock solution of BAAm was prepared by dissolving $0.3389 \mathrm{~g}$ of BAAm in $25 \mathrm{~mL}$ of distilled water.
Polymerization. The nanocomposite hydrogels were prepared by free-radical polymerization of AAm in aqueous suspensions of Laponite RDS with or without BAAm at $25{ }^{\circ} \mathrm{C}$. The initial concentration of the monomer AAm was set to $5 \% \mathrm{w} / \mathrm{v}$ while the Laponite concentration, $c$, was varied between 0 and $9 \% \mathrm{w} / \mathrm{v}$. The reactions were carried out at three different chemical cross-linker ratios $X$, the molar ratio of the chemical cross-linker BAAm to the monomer AAm, namely at $X=0$ (no BAAm), $1 / 80$, and $1 / 250$. The reaction time was set to $24 \mathrm{~h}$. APS $(3.51 \mathrm{mM})$ and TEMED $(0.25 \mathrm{~mL} / 100 \mathrm{~mL}$ reaction solution) were used as the redox initiator system. To illustrate the synthetic procedure, we give details for the preparation of hydrogels at $c=1.1 \% \mathrm{w} / \mathrm{v}$ and $X=0$ :

AAm $(0.5 \mathrm{~g})$ was dissolved in $8 \mathrm{~mL}$ of a $1.375 \% \mathrm{w} / \mathrm{v}$ Laponite RDS suspension, and then a stock solution of TEMED (1 mL) was added. The solution was purged with nitrogen gas for $10 \mathrm{~min}$, and APS stock solution $(1 \mathrm{~mL})$ was then added. For the rheological experiments, a portion of this solution was transferred between the parallel plates of the rheometer. For the swelling measurements, the solution was poured into several glass tubes of $4 \mathrm{~mm}$ i.d. and about $100 \mathrm{~mm}$ long. The glass tubes were sealed, and the polymerization was conducted for 1 day at $25{ }^{\circ} \mathrm{C}$. For the light scattering measurements, the solution was filtered through Nylon membrane filters with a pore size of $0.2 \mu \mathrm{m}$ and transferred in light scattering vials.

Rheological Experiments. Gelation reactions were carried out between the parallel plates of the rheometer (Gemini 150 rheometer system, Bohlin Instruments) equipped with a Peltier device for temperature control. The upper plate (diameter $40 \mathrm{~mm}$ ) was set at a distance of $500 \mu \mathrm{m}$ before the onset of the reactions, i.e., during the induction period. During all rheological measurements, a solvent trap was used to minimize the evaporation. A frequency of $\omega=1$ $\mathrm{Hz}$ and a deformation amplitude $\gamma^{\circ}=0.01$ were selected to ensure that the oscillatory deformation is within the linear regime. The reactions were monitored in the rheometer at $25^{\circ} \mathrm{C}$ up to a reaction time of about $3 \mathrm{~h}$ to avoid the effect of the solvent evaporation. Thereafter, frequency-sweep tests at $\gamma^{\circ}=0.01$ were carried out over the frequency range $0.1-10 \mathrm{~Hz}$.

Swelling Measurements. The nanocomposite hydrogels formed in glass tubes of $4 \mathrm{~mm}$ i.d. were cut into samples of about $10 \mathrm{~mm}$ length. Then, each sample was placed in an excess of water at $25^{\circ} \mathrm{C}$. In order to reach swelling equilibrium, the hydrogels were immersed in water for 1 month, replacing water several times. The swelling equilibrium was tested by weighing the gel samples. To achieve good precision, five measurements were carried out on samples of different length taken from the same gel. Swelling measurements were also carried out in aqueous $\mathrm{NaCl}$ solutions of concentrations ranging from $10^{-5}$ to $1.0 \mathrm{M}$. The hydrogels equilibrium swollen in water were transferred to vials containing the most diluted aqueous $\mathrm{NaCl}$ solution. The gel samples were allowed to swell in the solution for 2 weeks, during which aqueous $\mathrm{NaCl}$ was refreshed to keep the concentration as needed. After the swelling equilibrium is established, the gel samples were transferred into the next concentrated $\mathrm{NaCl}$ solution. The interpretation of the swelling measurements was made on the basis of the relative weight swelling ratio of gels, $m_{\text {rel }}$, which was calculated as $m_{\text {rel }}=m / m_{0}$, where $m$ is the mass of the equilibrium swollen gel sample in water or in $\mathrm{NaCl}$ solution and $m_{0}$ is its mass after preparation. It should be noted that, after equilibrium swelling of the hydrogels in water or in aqueous salt solutions and subsequent drying, the initial mass of AAm and Laponite remained unchanged. This indicates that neither PAAm nor nanoparticles are extracted from the gel network during the swelling tests.

Dynamic Light Scattering (DLS) Measurements. DLS measurements were performed at $25^{\circ} \mathrm{C}$ after a reaction time of $24 \mathrm{~h}$ using an ALV/CGS-3 compact goniometer (ALV, Langen, Germany) equipped with a cuvette rotation/translation unit (CRTU) and a $\mathrm{He}-\mathrm{Ne}$ laser $(22 \mathrm{~mW}$, wavelength $\lambda$ of $632.8 \mathrm{~nm})$. The scattering angle was fixed to be $90^{\circ}$. A fiber optical detection unit based on three-mode detection was used, which includes an appropriate collimator/GRIN-lens fiber and the ALV/STATIC and DYNAMIC enhancer. Ideally, the three-mode detection unit gives 
an intercept in the time-averaged intensity autocorrelation function (ICF) $g_{T}^{(2)}(q, 0)-1$ of 0.33 ; i.e., $\beta$ should be 0.33 . An experimental check with a polystyrene latex suspension gave a coherence factor $\beta$ of $0.36 \pm 0.01$, and this value was used for the evaluation of the DLS measurements. To protect the detector, the intensity of the incident light is automatically attenuated at each measurement by an eight-step automatic software-controlled attenuator and measured with a monitor diode. Thus, the intensity of incident light can be different within a series of measurements. When discussing scattering intensities, we therefore use data that were rescaled to a preset value of the monitor diode assuming a linear count rate dependence. Toluene was used as the index matching liquid. The temperature was controlled with an external thermostat. The timeaveraged ICFs were acquired at 100 different sample positions selected by randomly moving the CRTU before each run. The acquisition time for each run was $30 \mathrm{~s}$. The ensemble-averaged scattering intensity, $\langle I(q)\rangle_{\mathrm{E}}$, was determined by continuously moving the sample vial with the CRTU, and the acquisition time for $\langle I(q)\rangle_{\mathrm{E}}$ was 3 min.

Static Light Scattering Measurements. For the static light scattering measurements from Laponite dispersions, the scattered light intensities were measured at $25{ }^{\circ} \mathrm{C}$ using a commercial multiangle light scattering DAWN EOS (Wyatt Technologies Corp.) equipped with a vertically polarized $30 \mathrm{~mW}$ gallium arsenide laser operating at $\lambda=690 \mathrm{~nm}$ and 18 simultaneously detected scattering angles. The light scattering system was calibrated against a toluene standard (Rayleigh ratio at $690 \mathrm{~nm}=9.78 \times 10^{-6} \mathrm{~cm}^{-1}$, DAWN EOS software). Dispersions of Laponite with concentration in the range of $0.2-4.5 \% \mathrm{w} / \mathrm{v}$ in water were prepared from dry Laponite RDS powder. The scattered light intensities were recorded from angles $\theta=14.5^{\circ}$ to $152.5^{\circ}$, which correspond to the scattering vector $q$ range $3.1 \times 10^{-4}-2.4 \times 10^{-3} \AA^{-1}$, where $q=(4 \pi n / \lambda)$ $\sin (\theta / 2)$ and $n$ is the refractive index of the medium. The refractive index increment $\mathrm{d} n / \mathrm{d} c$ was determined at $25^{\circ} \mathrm{C}$ using the same Laponite dispersions using DAWN Optilap DSP at $690 \mathrm{~nm}$. Before the measurements, the system was calibrated using $\mathrm{NaCl}$ solutions. The $\mathrm{d} n / \mathrm{d} c$ value of the Laponite dispersion was found to be 0.083 \pm 0.002 .

\section{Results and Discussion}

Laponite Dispersions. In the present work, we used Laponite RDS, a Laponite clay modified with pyrophosphate ions. The edges of Laponite RDS are negatively charged so that the surfaces and edges of disks exhibit the same charge, leading to electrostatic repulsion among the disks and efficiently prohibiting the formation of a gel structure. ${ }^{23,24}$ Indeed, Laponite RDS can be easily dispersed in water up to about $10 \%$ concentration. When Laponite RDS powder is added to water and vigorously shaken, an initially opaque suspension is formed, and at concentrations below 5\% w/v Laponite, the suspension becomes completely transparent in a few hours. Thereafter, further development of the dispersion process can be monitored by measuring the scattered light intensity from the dispersions. Figure 1A shows the scattered light intensity in terms of the Rayleigh ratio $R(q)$ plotted against the square of the scattering vector $q$ for a $4.5 \% \mathrm{w} / \mathrm{v}$ Laponite dispersion at various mixing times. It is seen that a strong upturn at low $q$ appears at short mixing times, indicating the presence of a small fraction of large agglomerates. However, at longer times, the upturn disappears and $R(q)$ becomes almost independent of the scattering vector $q$. The results suggest that, although the dispersion becomes transparent in a few hours, the agglomerates of the Laponite crystals disappear only slowly, and finally, a stable suspension of the dispersed Laponite crystals is formed. It should be noted that the mixing times given in the caption to Figure 1A strongly depend on the rate and type of mixing. The data in Figure 1A were obtained by shaking the Laponite-water mixture twice a
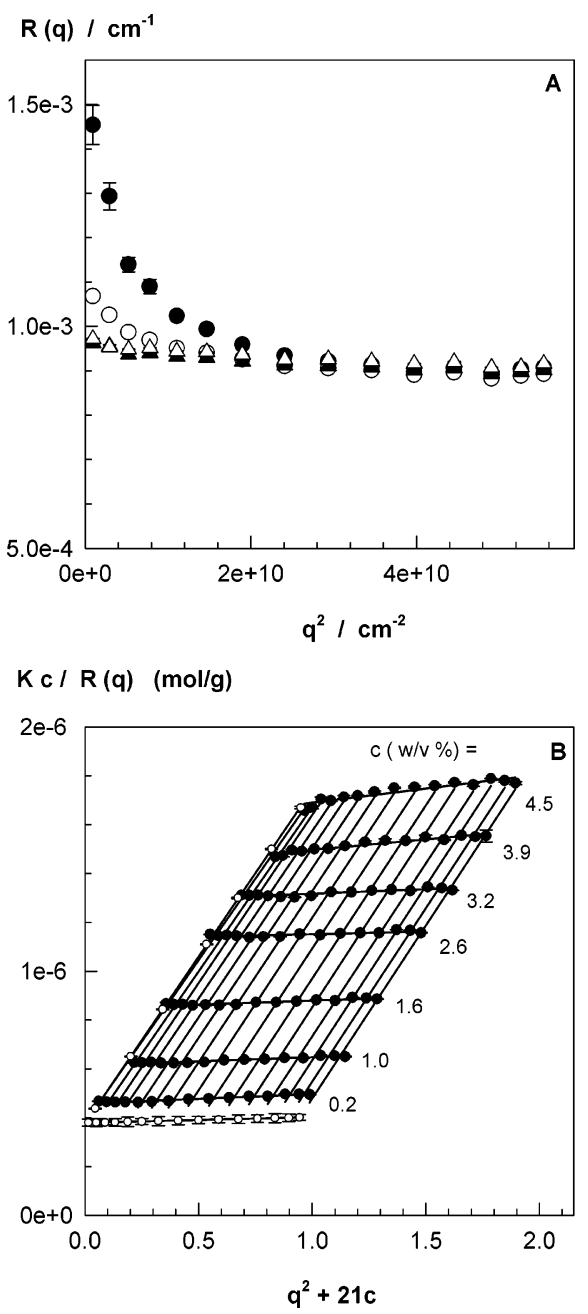

Figure 1. (A) Scattered light intensity in terms of the Rayleigh ratio $R(q)$ plotted against the square of the scattering vector $q$ for a $4.5 \%$ w/v Laponite dispersion at various mixing times. Mixing times: 4 (filled circle), 7 (open circle), 14 (filled triangle), and 19 days (open triangle). (B) Zimm plot of aqueous Laponite dispersions. Laponite concentrations are indicated. Open symbols are extrapolations to zero concentrations or zero scattering angles.

day; if the mixture is shaken continuously, one obtains $q$ independent Rayleigh ratios within 1 day.

After the scattered light intensity from the Laponite dispersion levels off at long mixing times, the scattering data were recorded at various scattering vectors $q$ and Laponite concentrations $c$. The results are shown in Figure 1B in the form of a Zimm plot according to the equation

$$
\frac{K c}{R(q)}=\frac{1}{\bar{M}_{\mathrm{w}}}\left(1+\frac{R_{\mathrm{g}}{ }^{2} q^{2}}{3}-\ldots\right)+2 A_{2} c+\ldots
$$

where $R_{\mathrm{g}}$ is the radius of gyration of the Laponite particles, $A_{2}$ is the second virial coefficient, $\bar{M}_{\mathrm{w}}$ is the weight-average molecular weight of the particles, and $K$ is an optical constant given by

$$
K=\frac{4 \pi^{2} n^{2}(\mathrm{~d} n / \mathrm{d} c)^{2}}{N_{\mathrm{A}} \lambda^{4}}
$$

where $N_{\mathrm{A}}$ is Avogadro's number. From the Zimm plot, the radius of gyration and the weight-average molecular weight were determined to be $20 \pm 2 \mathrm{~nm}$ and $(2.5 \pm 0.3) \times 10^{6} \mathrm{~g} / \mathrm{mol}$, respectively. These values are close to the reported values in 


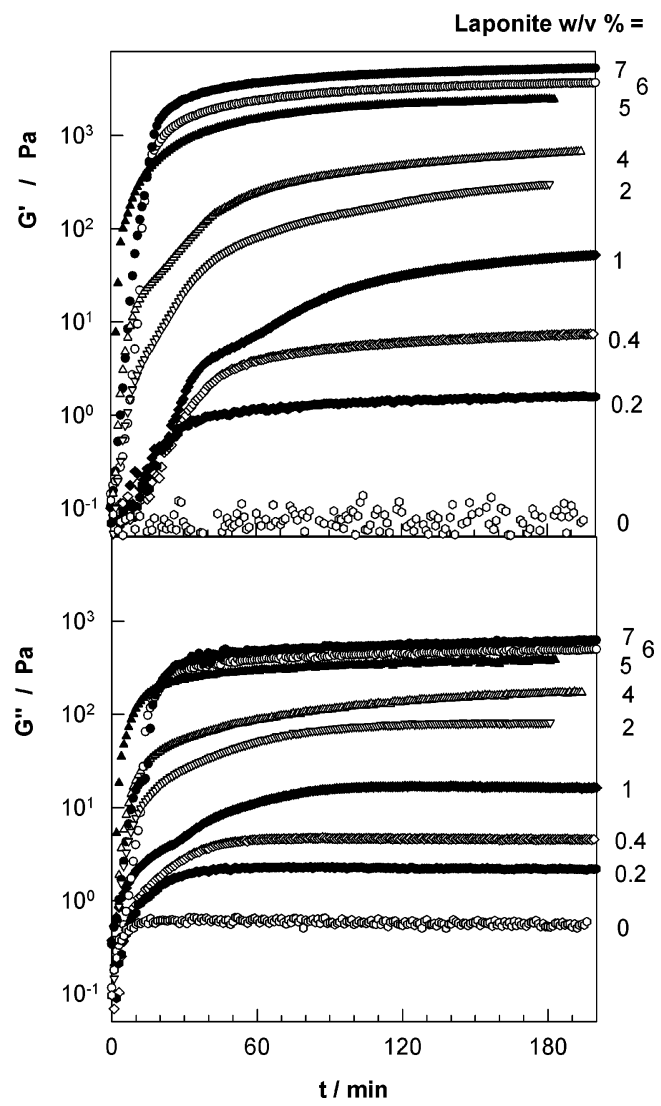

Figure 2. Elastic moduli $G^{\prime}$ and the viscous moduli $G^{\prime \prime}$ (measured at $1 \mathrm{~Hz}$ ) during the AAm polymerization in the presence of Laponite shown as a function of the reaction time $t . X=0$. Laponite concentrations are shown in the figure.

the literature. ${ }^{18,25-28}$ Thus, light scattering measurements indicate that Laponite disperses in water into a distribution of individual particles and small oligomers.

Rheological Monitoring of Nanocomposite Gel Formation. After formation of stable Laponite dispersions, the monomer AAm and the redox initiator system APS/TEMED were added into the dispersion, and the polymerization was started at $25{ }^{\circ} \mathrm{C}$. The polymerization reactions of AAm in aqueous Laponite dispersions and the formation of nanocomposite hydrogels were monitored by rheometry. By all the experiments, a certain induction period was observed, i.e., an initial reaction period during which the moduli are below the detection level. The duration of the induction period was found to vary between 3 and $10 \mathrm{~min}$. However, repeated measurements showed that the results are unaffected from these variations. In the following, the reaction time is defined as the total time elapsed minus the time needed for the induction period. In Figure 2, the elastic moduli $G^{\prime}$ and $G^{\prime \prime}$ measured at $1 \mathrm{~Hz}$ are shown as a function of the reaction time $t$. Semilogarithmic plots were chosen for clearer representation of the changes in the moduli of the reaction system at short reaction times. The Laponite concentrations are shown in the figure. During the AAm polymerization in the presence of Laponite, both moduli increase rapidly and then approach plateau values after $1-2 \mathrm{~h}$. The addition of just a small amount of Laponite into the monomer solution already leads to a marked increase of both $G^{\prime}$ and $G^{\prime \prime}$, suggesting strong interactions of PAAm chains with these nanoparticles.

Figure 3 shows the effect of the chemical cross-linker BAAm on the elastic and viscous properties of the nanocomposite hydrogels. Here, $G^{\prime}$ and $G^{\prime \prime}$ are shown as a function of the reaction time $t$ for three different cross-linker ratios $X$ used (mole chemical cross-linker BAAm/mol monomer AAm). $X=0$ corresponds to the polymerization of AAm in the absence of BAAm, and the data given in Figure 2 were redrawn for comparison. With or without the chemical cross-linker BAAm, the general trend is a rapid increase of the modulus followed by a plateau regime where the modulus slightly increases. The reaction period during which the modulus rapidly increases corresponds to the regime of the sol-gel transition as well as of the gel growth, while the slight increase corresponds to the post-cross-linking reactions in gel. ${ }^{29}$ Figure 3 also shows that, at a given Laponite concentration, the higher the BAAm content, the higher the elastic modulus $G^{\prime}$, but the lower the viscous modulus $G^{\prime \prime}$. Thus, addition of a chemical cross-linker into the gelation system decreases the viscous, energy dissipating properties of the nanocomposite materials.

After a reaction time of $3 \mathrm{~h}$, frequency-sweep tests at $\gamma^{\circ}=$ 0.01 were carried out over the frequency range $0.1-10 \mathrm{~Hz}$. Figure 4 shows the frequency dependence of $G^{\prime}$ (filled symbols) and $G^{\prime \prime}$ (open symbols) for the nanocomposite hydrogels of various Laponite contents and at a chemical cross-linker ratio $X=0$ (upper graphs) and 1/80 (lower graphs). Some $G^{\prime}$ values at low frequency were too small to be measured accurately. The pure PAAm solution shows a liquidlike response typical for a semidilute polymer solution, i.e., $G^{\prime \prime}$ exceeds $G^{\prime}$ at low frequencies, and there is a crossover between $G^{\prime}$ and $G^{\prime \prime}$ at a high frequency. In the presence of Laponite, however, $G^{\prime}$ becomes larger than $G^{\prime \prime}$ and shows a plateaulike behavior in the low-frequency range. This indicates the formation of viscoelastic gels. The height of the plateau increases and its width expands with increasing concentration of Laponite. At $7 \%$ Laponite, $G^{\prime}$ is almost independent of frequency while $G^{\prime \prime}$ remains on a fairly high level less than 1 order of magnitude smaller than $G^{\prime}$. The situation changes drastically in the presence of the chemical cross-linker BAAm (lower graphs). In this case, $G^{\prime}$ dominates over $G^{\prime \prime}$ by about 2 orders of magnitude over the entire range of Laponite concentration and is independent of frequency. This indicates formation of strong gels using the BAAm cross-linker. $G^{\prime \prime}$ values show some scatter due to the sensitivity limit of the rheometer in the determination of the phase angle $\delta$.

The gelation process is assumed to be complete when $G^{\prime}$ and $G^{\prime \prime}$ reach a plateau value with time. To estimate the final modulus of the gels, $G_{\infty}^{\prime}$, the experimental time-dependent modulus data $G^{\prime}$ shown in Figure 3 were fitted to a modified Hill equation: ${ }^{29-31}$

$$
G^{\prime}(t)=G_{\infty}^{\prime} \frac{t^{n}}{t^{\mathrm{n}}+\theta^{\prime n}}
$$

where $\theta^{\prime}$ is the time for which $G^{\prime}\left(\theta^{\prime}\right)=G^{\prime}{ }_{\infty} / 2$, i.e., the halfgelation time, and the exponent $n$ relates to the asymptotic slope $P$ at the half-gelation time $\theta^{\prime}$ by the following equation:

$$
P=\frac{n G_{\infty}^{\prime}}{4 \theta^{\prime}}
$$

The fitting results using eq 4 are also shown in Figure 3 by the solid curves. Half-gelation times $\theta^{\prime}$ decrease, i.e., the crosslinking reactions are promoted, by increasing the concentration of Laponite or BAAm (Figures 1A and 1B, Supporting Information). Further, the coefficient $n$ of eq 4 decreases with rising Laponite concentration, starting from 2 and approaching unity at $7 \%$ w/v Laponite (Figure 1C, Supporting Information). The extrapolated values of $G_{\infty}^{\prime}$ extracted from the fits are shown 

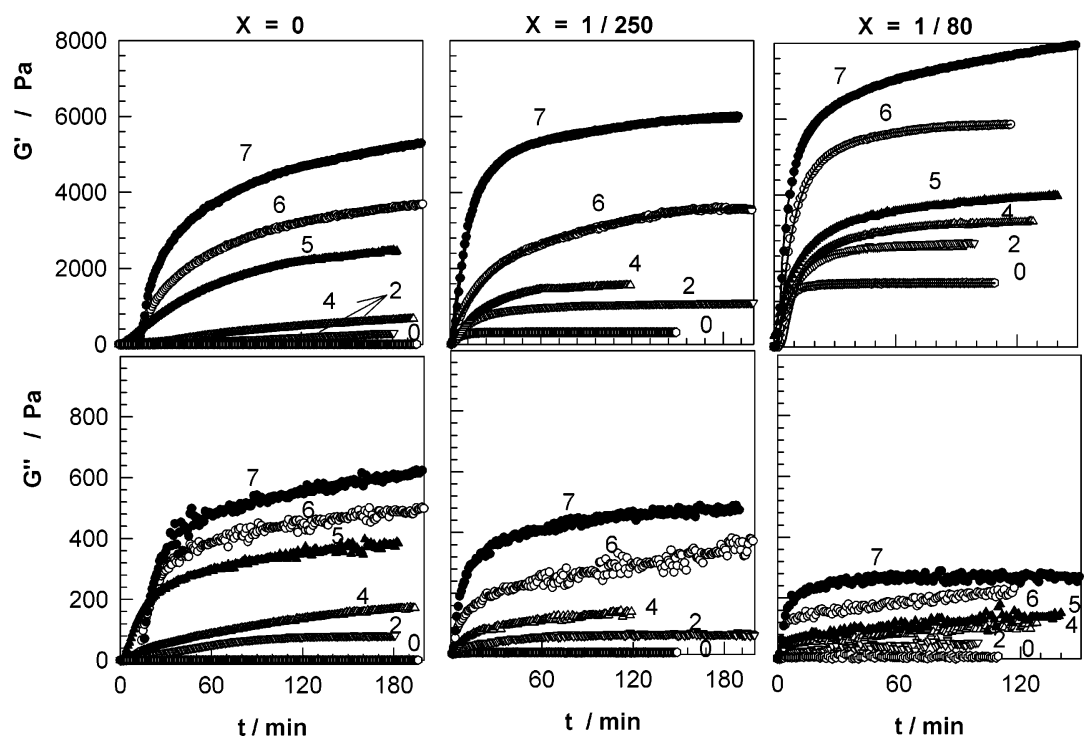

Figure 3. Elastic moduli $G^{\prime}$ and the viscous moduli $G^{\prime \prime}$ of the reaction system shown as a function of the reaction time $t$. The cross-linker ratio $X$ and the Laponite concentrations (in \% w/v) are indicated. The solid curves were calculated using eq 4.

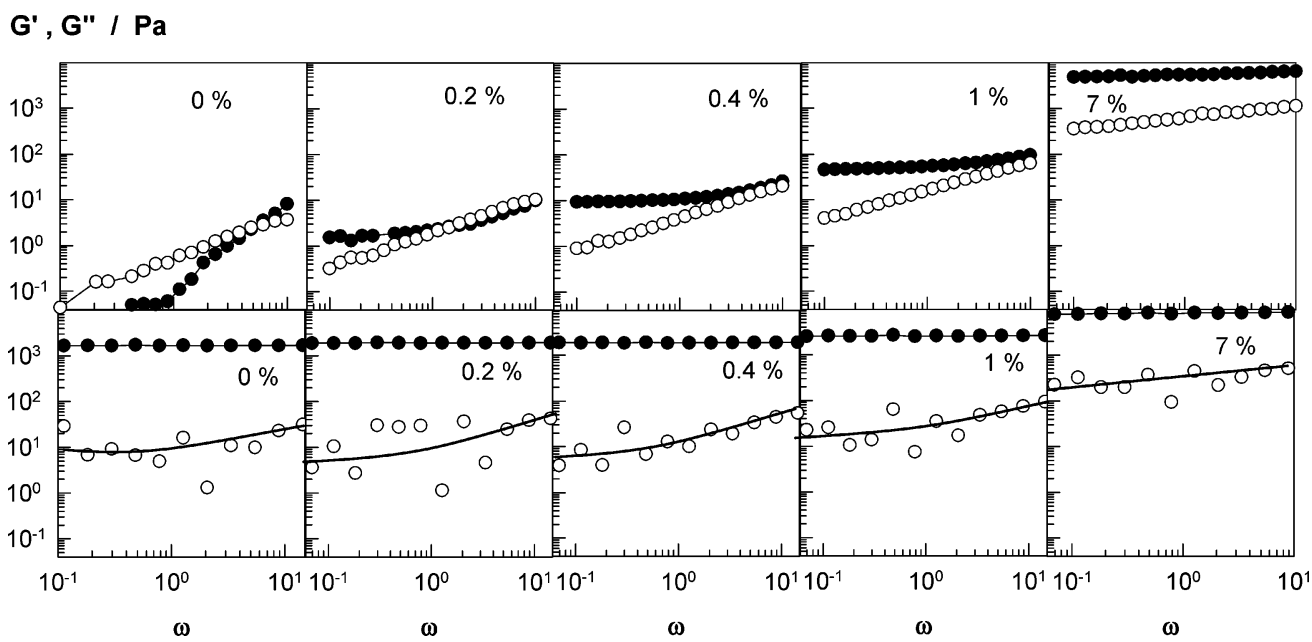

Figure 4. Elastic moduli $G^{\prime}$ (filled symbols) and viscous moduli $G^{\prime \prime}$ (open symbols) shown as a function of the frequency $\omega$ measured after $3 \mathrm{~h}$ of reaction time. $X=0$ (upper graphs) and 1/80 (lower graphs). Laponite concentrations are indicated. The solid curves are guides to the eye.

in Figure 5A as a function of the Laponite concentration. The limiting values of the viscous moduli $G^{\prime \prime}$ for each Laponite and BAAm concentration were also estimated from which the loss factor $\tan \delta$, which is the ratio of $G^{\prime \prime}$ to $G^{\prime}$, was calculated. These values are shown in Figure $5 \mathrm{~B}$ plotted against the Laponite concentration. Several interesting features can be seen from Figure 5A,B. First, in the absence of BAAm, increasing the Laponite concentration from 0.2 to $7 \%$ results in 3 orders of magnitude increase of the elastic modulus of the hydrogel. The marked increase in $G^{\prime}$ with the Laponite concentration suggests the existence of strong interactions between PAAm chains and clay so that the nanoparticles are fixed in the gel network as effective junction points. Thus, a PAAm hydrogel with an elastic modulus of the order of $10^{1} \mathrm{kPa}$ can be prepared using Laponite particles without a chemical cross-linker. In the presence of BAAm, the moduli of elasticity also increase with the Laponite concentration. At a Laponite concentration of 5\% or above, all systems, with or without the chemical cross-linker BAAm, exhibit similar elastic moduli, indicating that Laponite mainly determines the rubber elasticity of these hydrogels.

Since the final elastic modulus $G_{\infty}^{\prime}$ corresponds to the equilibrium shear modulus $G$, one may calculate the effective cross-link density $v_{\mathrm{e}}$ of the hydrogels as well as the average functionality $f_{\mathrm{e}}$ of the clay particles. Assuming phantom network behavior, $G$ at the state of gel preparation is given by 32,33

$$
G=\left(1-2 / f_{\mathrm{e}}\right) v_{\mathrm{e}} R T v_{2}^{0}
$$

where $v_{2}^{0}$ is the volume fraction of cross-linked polymer in the gel and $R$ and $T$ have their usual meanings. Furthermore, the functionality $f_{\mathrm{e}}$ of the nanoparticles can be estimated from the molecular weight $\bar{M}_{\mathrm{w}}$ of Laponite as ${ }^{18}$

$$
f_{\mathrm{e}}=2 v_{\mathrm{e}} v_{2}^{0} \bar{M}_{\mathrm{w}} / c
$$

where $c$ is the Laponite concentration in the hydrogel. Using $\bar{M}_{\mathrm{w}}=2.5 \times 10^{6} \mathrm{~g} / \mathrm{mol}$ and the modulus data given in Figure $5 \mathrm{~A}$ for the nanocomposite hydrogels with $X=0$ together with eqs 5 and 6 , we calculated $v_{\mathrm{e}}$ and $f_{\mathrm{e}}$ of the hydrogels prepared without the chemical cross-linker BAAm. For calculations, we assumed complete conversion of the monomer AAm to the cross-linked polymer. The filled circles in Figure 6 show the values of $v_{\mathrm{e}}$ of the nanocomposite hydrogels plotted against the Laponite concentration. As the Laponite concentration is increased from 0.4 to $7 \%$, the effective cross-link density $v_{\mathrm{e}}$ also increases from 0.2 to $70 \mathrm{~mol} / \mathrm{m}^{3}$. At the same time, the 


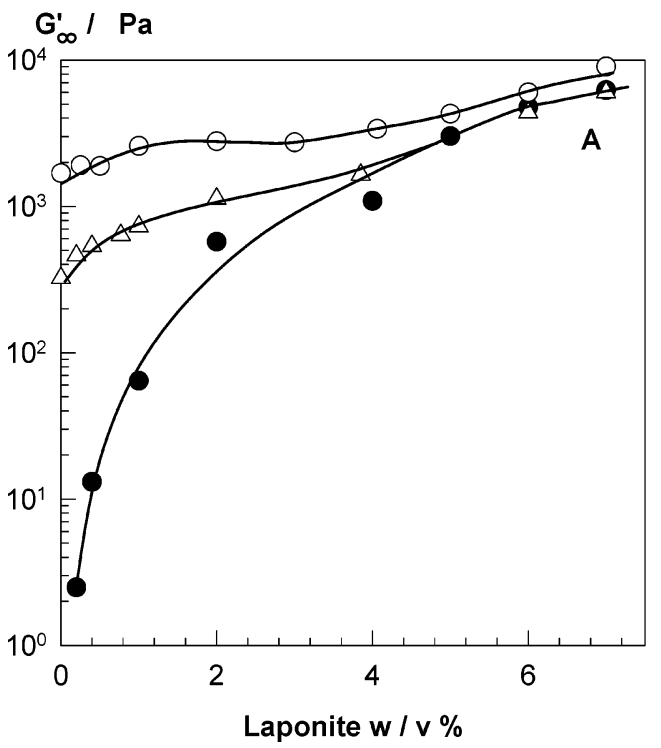

$\tan \delta$

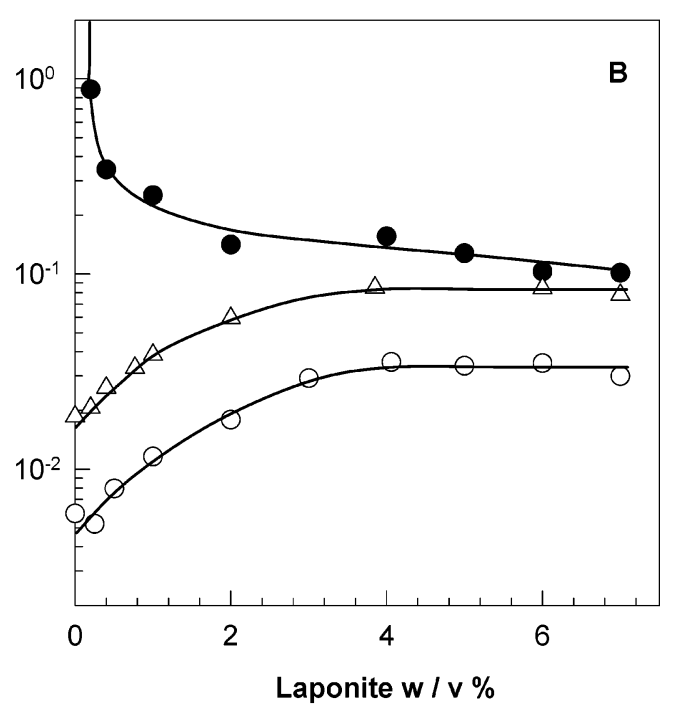

Figure 5. Final modulus of the gels $G_{\infty}^{\prime}$ and the loss factor $\tan \delta$ (both at $1 \mathrm{~Hz}$ ) shown as a function of the Laponite concentration. The cross-linker ratio $X=0$ (filled circle), 1/250 (open triangle), and 1/80 (open circle). The solid curves are guides to the eye.

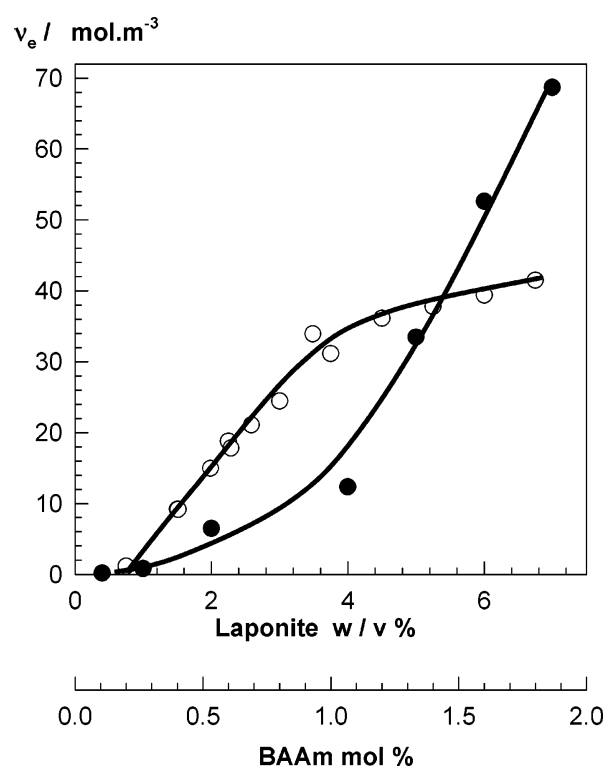

Figure 6. Effective cross-link density $v_{\mathrm{e}}$ of the nanocomposite hydrogels (filled circles) as a function of the Laponite concentration, and $v_{\mathrm{e}}$ of chemically cross-linked PAAm hydrogels (open symbols), calculated from the moduli data given in the literature. ${ }^{34}$ The solid curves are guides to the eye.

functionality $f_{\mathrm{e}}$ (not shown) of the nanoparticles, that is, the number of elastically effective network chains per clay particle, increases from 9 to 180, indicating that Laponite acts as a multifunctional cross-linker. It must be noted that the total number of PAAm chains bound to one Laponite particle may be much larger than $f_{\mathrm{e}}$ since an appreciable fraction of chains may not connect different clay particles and cannot store elastic energy upon deformation of the network. This portion of the chains will contribute to the viscous behavior of the hydrogels. For comparison, the effective cross-link densities $v_{\mathrm{e}}$ of chemically cross-linked PAAm hydrogels are also plotted in Figure 6 as a function of BAAm concentration (open symbols). These were calculated from the elastic moduli data given in ref 34 . Both sets of hydrogels were prepared at the same initial monomer concentration. While for the gels cross-linked by Laponite $v_{\mathrm{e}}$ increases only slightly up to $4 \%$ of clay and then more steeply with further rising clay concentration, the effective cross-link density of the chemically cross-linked hydrogels shows a strong initial rise but then levels off at higher BAAm contents. This typical behavior is due to the increasing extent of the multiple cross-linking reactions with increasing amount of BAAm, leading to the formation of clusters of cross-links. ${ }^{14}$ Thus, as the BAAm content in the comonomer feed is increased, more and more BAAm molecules are wasted in elastically ineffective links. On the other hand, the cross-linking efficiency of the nanoparticles rises as their concentration is increased. This is due to the simultaneously rising functionality.

As described above, from the limiting values of $G^{\prime}$ and $G^{\prime \prime}$, the loss factor $\tan \delta=G^{\prime \prime} / G^{\prime}$ was calculated for each polymerclay system (data measured at $1 \mathrm{~Hz}$ ). The quantity $\tan \delta$ represents the ratio of dissipated energy to stored energy during one deformation cycle. Figure 5B shows that, in the absence of the chemical cross-linker BAAm (filled symbols), $\tan \delta$ is below unity; that is, the reaction system shows a gel-like response even at a Laponite concentration of only $0.2 \%$. With increasing Laponite concentration, $\tan \delta$ decreases and approaches a limiting value of 0.1 at high concentrations. A relatively large frequency dependence of the moduli accompanied by a tan $\delta$ value $>0.1$ is a typical feature of a so-called weak gel. ${ }^{35,36}$ Thus, although the moduli of elasticity may reach to kilopascals, PAAm/Laponite nanocomposite hydrogels display characteristics of weak gels even at a high Laponite concentration. For the chemically cross-linked hydrogels without Laponite, $\tan \delta$ is around 0.01 ; i.e., $G^{\prime}$ is 2 orders of magnitude higher than $G^{\prime \prime}$ so that they are strong gels with negligible viscous properties. ${ }^{37}$ Interestingly, addition of Laponite particles into these gels leads to an increase in $\tan \delta$ (open symbols in Figure 5B), indicating that the viscous properties are raised more strongly than the elastic properties with increasing Laponite concentration.

One may speculate about the cause of the large energy dissipation occurring in the gels that contain Laponite. For the present polymer-clay system, one may consider that the PAAm chains are in a dynamic adsorption/desorption equilibrium with the clay particles. Thus, $\tan \delta$ represents the ratio of claypolymer adhesive bonds being broken down and reconstituted during dynamic strains to those remaining intact and unchanged. ${ }^{38}$ As an alternative, one may consider friction at the clay-polymer interface resulting from sliding of the adsorbed chains along the clay surfaces. Both processes would require 
an extensive rearrangement of the highly entangled polymer material close to the clay surface, which should proceed with long relaxation times and a broad relaxation time spectrum. An order of magnitude larger value of $\tan \delta$ of nanocomposite hydrogels compared to the chemically cross-linked hydrogels is an indication of such slow dynamic processes and results in the excellent mechanical performance of these materials reported before.

We assume that the mechanism suggested by Haraguchi is also applicable to the formation of the present polymer-clay systems with or without the chemical cross-linker BAAm. ${ }^{2-5}$ According to this mechanism, the monomers are bound to the surface of the clay particles due to hydrogen bonds between the oxygen atoms of clay and the amide protons of the monomers as well as due to complex formation between the metal ions on the clay surface and the carbonyl oxygen of the monomer. Since the monomer AAm is more hydrophilic than the cross-linker BAAm, the cross-linker ratio on the clay surface is expected to be lower than in the solution. Further, the initiator is also adsorbed on the clay surface by ionic interaction. ${ }^{2}$ After decomposition of the initiator and formation of primary radicals, the polymer chains start to grow mainly from the surface of the particles until they terminate by recombination with other polymeric radicals. These could be bound by their other end to the surface of other clay particles, resulting in the bridging of the particles and in the formation of effective network chains. Termination with unanchored radicals or termination by disproportionation leads to dangling chains. During the propagation step, the growing radicals also react with the cross-linker BAAm, leading to the formation of pendant vinyl groups. The reactions of these pendant vinyl groups with radical centers lead to the formation of effective chemical cross-links as well as of cycles and clusters of BAAm, as typical in cross-linking copolymerization of AAm and BAAm. However, as seen in Figure 5A, above $1 \%$ Laponite, the elastic modulus of the hydrogels is mainly determined by the amount of clay. Furthermore, with or without BAAm, the hydrogels exhibit a very strong dissipation mechanism compared to the chemically cross-linked gels, indicating that the dynamic adsorption/desorption equilibrium of the network chains with the particles dominates the elasticity of the hydrogels. Thus, the network chains between the clay particles rather than those between the BAAm molecules determine the rubber elasticity of the nanocomposite hydrogels.

Swelling Properties of Nanocomposite Hydrogels. The swelling capacities of the hydrogels were measured in water and in aqueous $\mathrm{NaCl}$ solutions. No chemical cross-linker was used in the hydrogel preparation. Figure 7A shows the relative weight swelling ratio $m_{\text {rel }}$ of the hydrogels in water plotted against the Laponite concentration. The hydrogels formed at or below 4\% Laponite were too weak to withstand the swelling tests. Therefore, only data in the range of 4.4-9.1\% Laponite are shown in the figure. It is seen that although the effective cross-link density significantly increases with the Laponite concentration (Figure 6), the equilibrium swelling ratio of the hydrogel in water also increases up to about $7 \%$ Laponite. This unusual effect is a consequence of the osmotic pressure exerted by the counterions of Laponite particles in the hydrogel. This osmotic pressure increases as the concentration of the counterions increases. Thus, the ionic character of Laponite dominates over its cross-linker effect so that increasing Laponite content induces gel swelling. A decrease in the swelling ratio appears with increasing amount of Laponite above $7 \%$, indicating that the cross-linker effect of the particles starts to dominate the swelling process. To demonstrate further the ionic character of

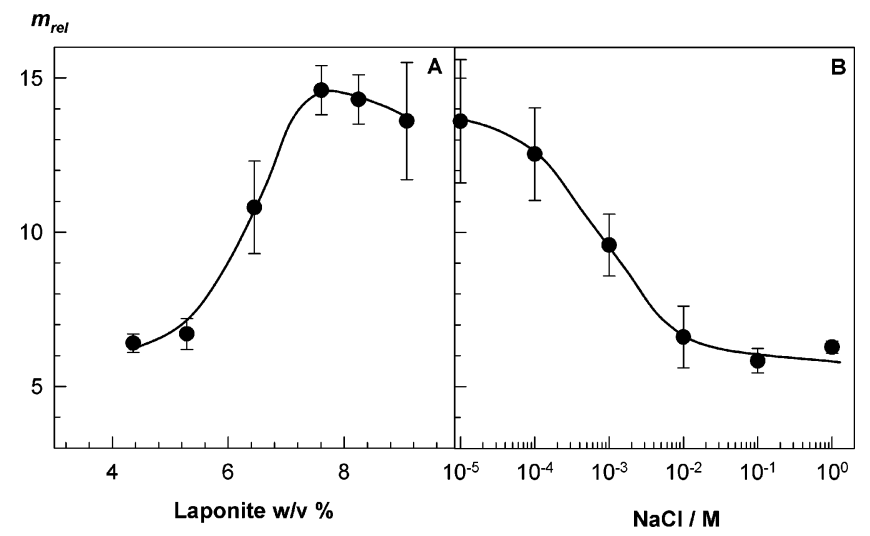

Figure 7. (A) Relative weight swelling ratio $m_{\text {rel }}$ of the hydrogels in water plotted against the Laponite concentration. $X=0$. (B) Relative weight swelling ratio $m_{\text {rel }}$ of the hydrogel with $9.1 \%$ Laponite shown as a function of $\mathrm{NaCl}$ concentration. $X=0$. The solid curves are guides to the eye.

the hydrogels, we investigated their swelling behavior in aqueous $\mathrm{NaCl}$ solutions. In Figure 7B, the relative weight swelling ratios $m_{\text {rel }}$ of the hydrogel with $9.1 \%$ Laponite are shown as a function of the salt concentration. Similar $m_{\text {rel }}$ vs salt concentration dependences were also obtained for other Laponite concentrations. Deswelling of the hydrogel with increasing salt concentration in the external solution is seen from the figure. This is due to the decrease in the concentration difference of counterions inside and outside the nanocomposite hydrogel and is typical behavior for ionic hydrogels. ${ }^{39}$

Dynamics of Nanocomposite Hydrogels. Dynamics of the nanocomposite hydrogels were systematically investigated by DLS measurements as a function of Laponite and BAAm concentrations. DLS provides the time-average intensity correlation function $g_{T}^{(2)}(q, \tau)$, defined as ${ }^{40}$

$$
g_{\mathrm{T}}^{(2)}(q, \tau)=\frac{\langle I(q, 0) I(q, \tau)\rangle_{T}}{\langle I(q, 0)\rangle_{T}{ }^{2}}
$$

whose short-time limit can be related to an apparent diffusion coefficient, $D_{\mathrm{A}}$, via ${ }^{40,41}$

$$
D_{\mathrm{A}}=-\frac{1}{2 q^{2}} \lim _{\tau \rightarrow 0} \ln \left(g_{\mathrm{T}}^{(2)}(q, \tau)-1\right)
$$

where $\tau$ is the decay time and $\langle\ldots\rangle_{T}$ denotes time average. For a nonergodic medium like polymer gels, $D_{\mathrm{A}}$ and, likewise, the time-averaged scattering intensity $\langle I(q)\rangle_{T}$ varies randomly with sample position. $\langle I(q)\rangle_{T}$ has two contributions: one from static inhomogeneities (frozen structure) and the other from dynamic fluctuations according to the following equation: ${ }^{40-42}$

$$
\langle I(q)\rangle_{T}=I_{\mathrm{C}}(q)+\left\langle I_{\mathrm{F}}(q)\right\rangle_{T}
$$

where $I_{\mathrm{C}}(q)$ and $\left\langle I_{\mathrm{F}}(q)\right\rangle_{T}$ are the scattered intensities due to the frozen structure and liquidlike concentration fluctuations, respectively. To separate $\langle I(q)\rangle_{T}$ into its two parts, we follow the method proposed by Joosten et al. ${ }^{40}$ Treating the system by the partial heterodyne approach, one obtains

$$
D=D_{\mathrm{A}} /\left(2-X_{\mathrm{p}}\right)
$$

where $D$ is the cooperative diffusion coefficient and $X_{\mathrm{p}}=$ $\left\langle I_{\mathrm{F}}(q)\right\rangle_{T} /\langle I(q)\rangle_{T}$. Equation 10 applies to each sample position. For different sample positions, different values of $D_{\mathrm{A}}$ and $\langle I(q)\rangle_{T}$ are obtained. Then, if many measurements at different sample 

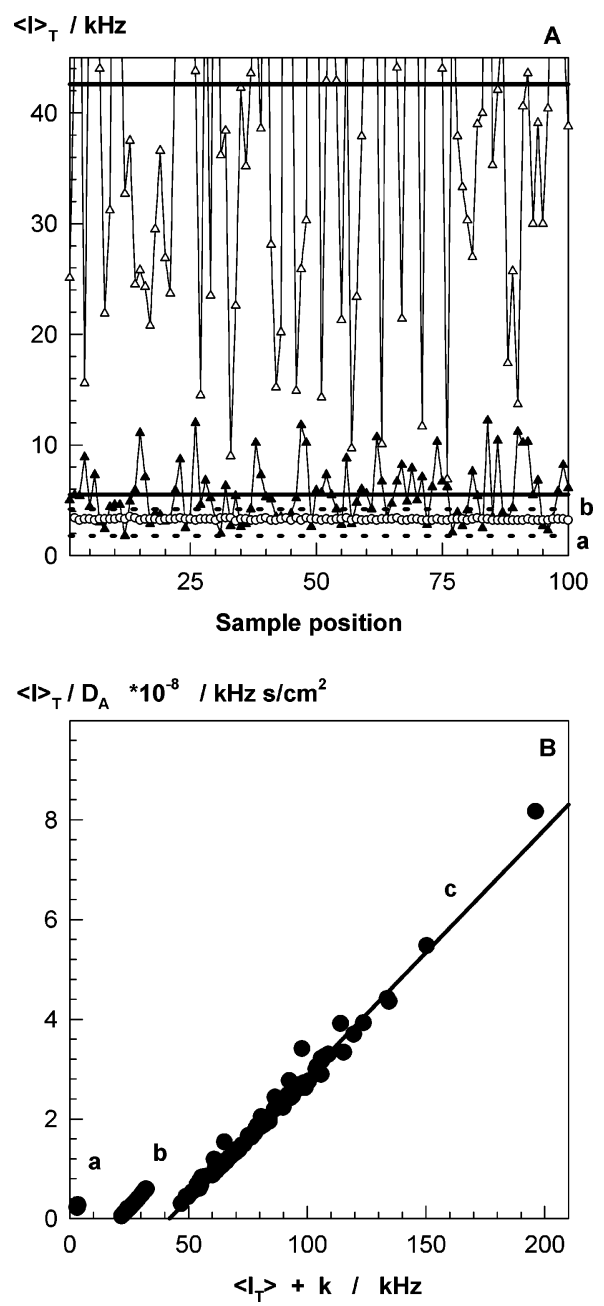

Figure 8. (A) Variation of the time-averaged scattered intensity $\langle I\rangle_{T}$ with the sample position for PAAm solution (open circles), for PAAm gel at a cross-linker ratio $X=1 / 80$ without and with Laponite particles of $c=0.27 \%$ (filled and open triangles, respectively). The solid lines represent the ensemble-averaged scattered intensity $\langle I\rangle_{\mathrm{E}}$. The fluctuating component of the scattered intensity $\left\langle I_{\mathrm{F}}\right\rangle_{T}$ for PAAm gels without and with Laponite are represented by the dotted lines a and b, respectively. (B) Decomposition plots according to eq 10a for PAAm solution (a) and for PAAm gel at a cross-linker ratio $X=1 / 80$ without and with Laponite particles of $c=0.27 \%$ (b and c, respectively). $k$ is the shift factor taken as 0,20 , and 40 for PAAm solution and PAAm gel without and with Laponite, respectively.

positions are performed, the cooperative diffusion coefficient $D$ and the fluctuating component of the scattering intensity $\left\langle I_{\mathrm{F}}(q)\right\rangle_{T}$ can be obtained by plotting $\langle I(q)\rangle_{T} / D_{\mathrm{A}}$ vs $\langle I(q)\rangle_{T}$ according to eq $10 \mathrm{a}$, which is simply a rearrangement of eq 10 .

$$
\frac{\langle I(q)\rangle_{T}}{D_{\mathrm{A}}}=\frac{2\langle I(q)\rangle_{T}}{D}-\frac{\left\langle I_{\mathrm{F}}(q)\right\rangle_{T}}{D}
$$

Figure $8 \mathrm{~A}$ shows the variations of time-averaged scattered intensity $\langle I\rangle_{T}$, measured at $\theta=90^{\circ}$, with the sample position for PAAm solution (open circles), for PAAm gel at a crosslinker ratio $X=1 / 80$ without and with Laponite particles of $c$ $=0.27 \%$ (filled and open triangles, respectively). The solid lines represent the ensemble-averaged scattered intensity, $\langle I\rangle_{\mathrm{E}}$, measured as described in the Experimental Part. As expected, $\langle I\rangle_{T}$ $=\langle I\rangle_{\mathrm{E}}$ for PAAm solution because a polymer solution is an ergodic medium. By introducing chemical cross-links, fluctuations in $\langle I\rangle_{T}$ appear. Such fluctuations of $\langle I\rangle_{T}$ with the sample position become much stronger if, in addition to BAAm, a small amount of Laponite is also included into the hydrogel. In order
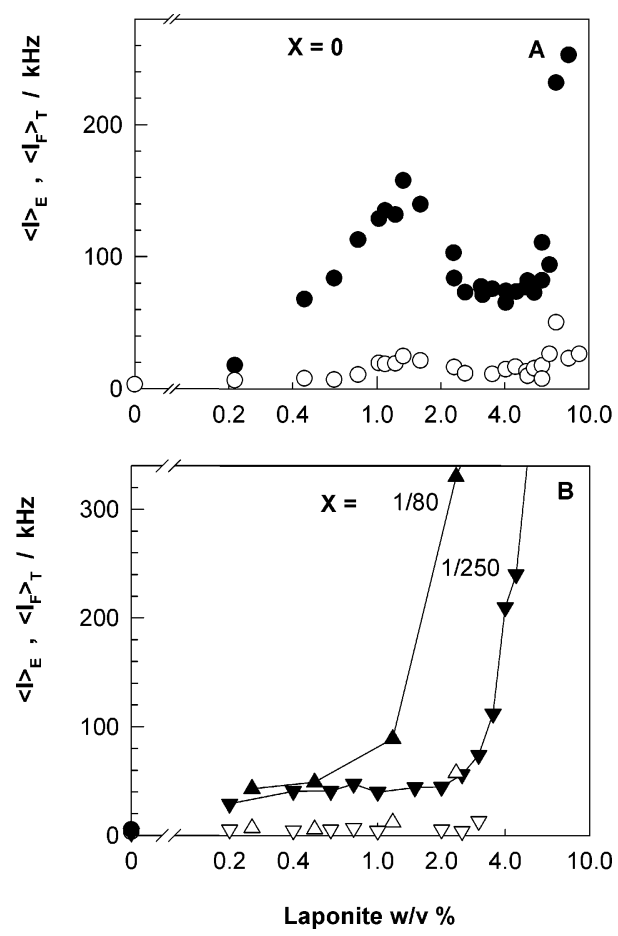

Figure 9. Variations of the ensemble-averaged scattered intensity $\langle I\rangle_{\mathrm{E}}$ (filled symbols) and the fluctuating component of the scattered intensity $\left\langle I_{\mathrm{F}}\right\rangle_{T}$ (open symbols) as a function of Laponite concentration without (A) and with the chemical cross-linker BAAm (B).

to extract the contribution of the dynamic fluctuation component of the scattered intensity, decomposition of $\langle I\rangle_{T}$ was carried out by using eq 10a. Figure $8 \mathrm{~B}$ shows examples of the decomposition plots for the three polymer systems given in Figure 8A. For each system 100 data points are plotted. For PAAm solution, all data points collapse to a single point due to the ergodicity of the system. From the linear regression analysis of PAAm gel with and without Laponite particles, diffusion coefficients as well as the fluctuating component of the scattered intensity $\left\langle I_{\mathrm{F}}\right\rangle_{T}$ were evaluated. In Figure $8 \mathrm{~A},\left\langle I_{\mathrm{F}}\right\rangle_{T}$ for PAAm gels with or without Laponite are represented by the dotted lines $b$ and a, respectively. It is seen that the main contribution to the scattered light intensity in nanocomposite hydrogel originates from frozen concentration fluctuations.

In Figure 9, the variations of the ensemble-averaged scattered intensity $\langle I\rangle_{\mathrm{E}}$ (filled symbols) and the fluctuating component of the scattered intensity $\left\langle I_{\mathrm{F}}\right\rangle_{T}$ (open symbols) are shown as a function of Laponite concentration with and without the chemical cross-linker BAAm. $\left\langle I_{\mathrm{F}}\right\rangle_{T}$ does not change significantly with the Laponite concentration. Thus, the magnitude of the ensemble-averaged scattered intensity $\langle I\rangle_{\mathrm{E}}$ also reflects the extent of the frozen concentration fluctuations due to the spatial gel inhomogeneity. Figure 9A shows that as soon as a gel is formed (between 0.2 and $0.4 \%$ Laponite), $\langle I\rangle_{\mathrm{E}}$ becomes larger than $\left\langle I_{\mathrm{F}}\right\rangle_{T}$ due to the nonergodicity of the system. $\langle I\rangle_{\mathrm{E}}$ increases up to $1 \%$ Laponite but then decreases and remains constant between 2.5 and $6 \%$ Laponite. At still higher Laponite concentrations, $\langle I\rangle_{\mathrm{E}}$ increases again rapidly, and the system becomes strongly opaque. Moreover, in the presence of BAAm (Figure 9B), $\langle I\rangle_{\mathrm{E}}$ does not change with the Laponite concentration up to a critical value but then rapidly increases.

The results thus obtained contradict previous observations regarding the cross-linker concentration dependence of the spatial gel inhomogeneity. Literature data clearly show a continuous increase in the degree of spatial inhomogeneity with the cross-linker concentration. ${ }^{12,13}$ The present results can be 
explained with the action of Laponite both as a cross-linker and as an ionic component during the formation of the nanocomposite hydrogels. Increasing Laponite concentration increases both the effective cross-link density and the concentration of mobile counterions $\left(\mathrm{Na}^{+}\right.$ions) in the hydrogel. While the inhomogeneity becomes larger due to the first effect, ${ }^{15}$ the latter effect decreases the gel inhomogeneity. ${ }^{43,44}$ The interplay of these two opposite effects seems to determine the spatial inhomogeneity of the nanocomposite hydrogels and results in a nonmonotonic variation of $\langle I\rangle_{\mathrm{E}}$ with the Laponite concentration. In the presence of the chemical cross-linker BAAm, the cross-linker effect of both Laponite and BAAm seems to dominate the inhomogeneity of gels so that the maximum shown in Figure 9A does not appear.

It should be noted that the experimental method used does not allow to explain the origin of the inhomogeneities in the present polymer-clay systems. The scattering intensity function for such ternary systems consisting of polymer, clay, and solvent is composed of polymer-polymer, clay-clay, and polymerclay structure factors. Recent contrast-matched SANS measurements show that the nanocomposite gels have very low crosslink inhomogeneities compared to the chemically cross-linked gels. $^{21,22}$ Thus, the origin of the spatial gel inhomogeneity in such hydrogels seems to differ from those of the chemically cross-linked gels. Indeed, as seen in Figure 8A, the degree of inhomogeneity is mainly determined by the amount of Laponite in the hydrogels. The inhomogeneity created due to the nonrandom cross-linking in AAm-BAAm network is markedly smaller. This indicates that the clay or polymer-clay scattering but not the polymer scattering determines the inhomogeneity as seen by light scattering in the present hydrogels.

\section{Conclusion}

The free-radical polymerization of acrylamide in aqueous clay dispersions and the structure of the resulting polymer-clay nanocomposite hydrogels have been investigated by rheometry using oscillatory deformation tests. Laponite with a radius of gyration in distilled water of $20 \mathrm{~nm}$ was used as clay particles in the hydrogel preparation. To understand the effect of Laponite on the rubber elasticity of the nanocomposite hydrogels, gelation reactions were carried out with and without the presence of the chemical cross-linker $N, N^{\prime}$-methylenebis(acrylamide) (BAAm). In the absence of BAAm, increasing clay concentration from 0.2 to $7 \%$ results in 3 orders of magnitude increase of the elastic modulus $G^{\prime}$ of the hydrogels. At a clay concentration of $5 \%$ or above, all the reaction systems, with or without BAAm, exhibit similar elastic moduli, indicating that clay mainly determines the rubber elasticity of the hydrogels. The rheological investigations also revealed that there exists a fundamental difference with regard to the loss factor tan $\delta$ between chemically crosslinked gels and those containing clay particles. The latter exhibit a markedly stronger dissipation mechanism ( $\tan \delta$ around 0.1 ), which is likely to be one reason for their outstanding mechanical properties. A large $\tan \delta$ in the frequency range inspected indicates long relaxation times and a broad relaxation time spectrum. This observation is complementary to the rather large correlation lengths found in similar systems. ${ }^{18}$ Dynamics of the nanocomposite hydrogels was also investigated by dynamic light scattering. The ensemble-averaged scattered intensity of the hydrogels varies nonmonotonically with the clay concentrations. The result was explained with the action of clay both as a crosslinker and as an ionic component during the formation of the nanocomposite hydrogels.
Acknowledgment. We are grateful to Alexander von Humboldt Stiftung for a grant to O. Okay. This work was also supported by the Scientific and Technical Research Council of Turkey (TUBITAK), TBAG-105T246.

Supporting Information Available: Figures showing the variations of half-gelation times $\theta^{\prime}$ and the coefficient $n$ of eq 4 as functions of clay and BAAm concentrations. This material is available free of charge via the Internet at http://pubs.acs.org.

\section{References and Notes}

(1) Bohidar, H. B., Dubin, P., Osada, Y., Eds.; Polymer Gels: Fundamentals and Applications; ACS Symposium Series 833; American Chemical Society: Washington, DC, 2002.

(2) Haraguchi, K.; Takehisa, T. Adv. Mater. 2002, 14, 1120-1124.

(3) Haraguchi, K.; Takehisa, T.; Fan, S. Macromolecules 2002, 35, 10162-10171.

(4) Haraguchi, K.; Farnworth, R.; Ohbayashi, A.; Takehisa, T. Macromolecules 2003, 36, 5732-5741.

(5) Haraguchi, K.; Li, H-J.; Matsuda, K.; Takehisa, T.; Elliott, E. Macromolecules 2005, 38, 3482-3490.

(6) Churochkina, N. A.; Starodoubtsev, S. G.; Khokhlov, A. R. Polym Gels Networks 1998, 6, 205-215.

(7) Liu, Y.; Zhu, M.; Liu, X.; Zhang, W.; Sun, B.; Chen, Y.; Adler, H.-J. P. Polymer 2006, 47, 1-5.

(8) Mallam, S.; Horkay, F.; Hecht, A. M.; Geissler, E. Macromolecules 1989, 22, 3356-3361.

(9) Cohen, Y.; Ramon, O.; Kopelman, I. J.; Mizraki, S. J. Polym. Sci., Polym. Phys. Ed. 1992, 30, 1055-1067.

(10) Schosseler, F.; Skouri, R.; Munch, J. P.; Candau, S. J. J. Phys. II 1994, 4, 1221-1239.

(11) Shibayama, M.; Tanaka, T.; Han, C. C. J. Chem. Phys. 1992, 97, 6842-6854.

(12) Shibayama, M. Macromol. Chem. Phys. 1998, 199, 1-30.

(13) Bastide, J.; Candau, S. J. In Physical Properties of Polymeric Gels; Cohen Addad, J. P., Ed.; Wiley: New York, 1996; p 143.

(14) Funke, W.; Okay, O.; Joos-Muller, B. Adv. Polym. Sci. 1998, 136, 139-234.

(15) Kizilay, M. Y.; Okay, O. Macromolecules 2003, 36, 6856-6862.

(16) Liu, R.; Oppermann, W. Macromolecules 2006, 39, 4159-4167.

(17) Lindemann, B.; Schröder, U. P.; Oppermann, W. Macromolecules 1997, 30, 4073-4077.

(18) Nie, J.; Du, B.; Oppermann, W. Macromolecules 2005, 38, 57295736.

(19) Shibayama, M.; Suda, J.; Karino, T.; Okabe, S.; Takehisa, T.; Haraguchi, K. Macromolecules 2004, 37, 9606-9612.

(20) Nie, J.; Du, B.; Oppermann, W. J. Phys. Chem. B 2006, 110, 1116711175.

(21) Shibayama, M.; Karino, T.; Miyazaki, S.; Okabe, S.; Takehisa, T.; Haraguchi, K. Macromolecules 2005, 38, 10772-10781.

(22) Miyazaki, S.; Karino, T.; Endo, H.; Haraguchi, K.; Shibayama, M. Macromolecules 2006, 39, 8112-8120.

(23) Mongondry, P.; Tassin, J.-F.; Nicolai, T. J. Colloid Interface Sci. 2005, 283, 397-405.

(24) Mongondry, P.; Nicolai, T.; Tassin, J.-F. J. Colloid Interface Sci. 2004, 275, 191-196.

(25) Avery, R. G.; Ramsay, J. D. F. J. Colloid Interface Sci. 1986, 109, 448-454.

(26) Rosta, L.; von Gunten, H. R. J. Colloid Interface Sci. 1990, 134, 397406.

(27) Nicolai, T.; Cocard, S. Langmuir 2000, 16, 8189-8193.

(28) Bhatia, S.; Barker, J.; Mourchid, A. Langmuir 2003, 19, 532-535.

(29) Calvet, D.; Wong, J. Y.; Giasson, S. Macromolecules 2004, 37, 77627771.

(30) Giraldo, J.; Vivas, N. M.; Vila, E.; Badia, A. Pharmacol. Therap. 2002, 95, 21-45.

(31) Hill, A. V. Biochem. J. 1913, 7, 471-480.

(32) Flory, P. J. Principles of Polymer Chemistry; Cornell University Press: Ithaca, NY, 1953.

(33) Treloar, L. R. G. The Physics of Rubber Elasticity; Oxford University Press: Oxford, 1975.

(34) Orakdogen, N.; Okay, O. Polym. Bull. (Berlin) 2006, 57, 631-641.

(35) Ross-Murphy, S. B.; Morris, V. J.; Morris, E. R. Faraday Symp. Chem. Soc. 1983, 18, 115-129.

(36) Ross-Murphy, S. B. J. Rheol. 1995, 39, 1451-1463.

(37) Oppermann, W.; Rose, S.; Rehage, G. Br. Polym. J. 1985, 17, 175180.

(38) Vu, Y. T.; Mark, J. E.; Pham, L. H.; Engelhardt, M. J. Appl. Polym. Sci. 2001, 82, 1391-1403.

(39) Durmaz, S.; Okay, O. Polymer 2000, 41, 3693-3704. 
(40) Joosten, J. G. H.; Mccarthy, J. L.; Pusey, P. N. Macromolecules 1991 , 24, 6690-6699.

(41) Pusey, P. N.; van Megen, W. Physica A 1989, 157, 705-741.

(42) Ikkai, F.; Shibayama, M. Phys. Rev. Lett. 1999, 82, 49464949.
(43) Kizilay, M. Y.; Okay, O. Polymer 2003, 44, 5239-5250.

(44) Starodoubtsev, S. G.; Churochkina, N. A.; Khokhlov, A. R. Langmuir 2005, 16, 1529-1534.

MA062929V 